\title{
Principal bundles over a real algebraic curve
}

\author{
INDRANIL Biswas AND JaCQues HuRtubise
}

Let $X$ be a compact connected Riemann surface equipped with an anti-holomorphic involution $\sigma$. Let $G$ be a connected complex reductive affine algebraic group, and let $\sigma_{G}$ be a real form of $G$. We consider holomorphic principal $G$-bundles on $X$ satisfying compatibility conditions with respect to $\sigma$ and $\sigma_{G}$. We prove that the points defined over $\mathbb{R}$ of the smooth locus of a moduli space of principal $G$-bundles on $X$ are precisely these objects, under the assumption that genus $(X) \geq 3$. Stable, semistable and polystable bundles are defined in this context. Relationship between any of these properties and the corresponding property of the underlying holomorphic principal $G$-bundle is explored. A bijective correspondence between unitary representations and polystable objects is established.

\section{Introduction}

Let $X$ be a compact connected Riemann surface, and let

$$
\sigma: X \longrightarrow X
$$

be an anti-holomorphic involution. Let $G$ be a connected complex reductive linear algebraic group equipped with an anti-holomorphic involutive automorphism $\sigma_{G}$. The subgroup of the center of $G$ that is fixed by $\sigma$ will be denoted by $Z_{\mathbb{R}}$.

For a holomorphic principal $G$-bundle $E_{G}$ on $X$, let $\bar{E}_{G}$ be the corresponding $C^{\infty}$ principal $G$-bundle obtained by twisting the action of $G$ on $E_{G}$ using $\sigma_{G}$. The pullback $\sigma^{*} \bar{E}_{G}$ has a natural holomorphic structure.

A pseudo-real principal $G$-bundle on $X$ is defined to be a pair of the form $\left(E_{G}, \rho\right)$, where $E_{G}$ is a holomorphic principal $G$-bundle on $X$, and

$$
\rho: E_{G} \longrightarrow \sigma^{*} \bar{E}_{G}
$$


is a holomorphic isomorphism of principal $G$-bundles satisfying the condition that there is an element $c \in Z_{\mathbb{R}}$ such that the composition

$$
E_{G} \stackrel{\rho}{\longrightarrow} \sigma^{*} \bar{E}_{G} \stackrel{\sigma^{*} \bar{\rho}}{\longrightarrow} \sigma^{*} \bar{\sigma}^{*} \bar{E}_{G}=\sigma^{*} \sigma^{*} \overline{\bar{E}}_{G}=E_{G}
$$

coincides with the automorphism of $E_{G}$ given by $c$. (The details are in Section 2.1.) If $c=e$, then $\left(E_{G}, \rho\right)$ is called a real principal $G$-bundle.

We define semistable, stable and polystable pseudo-real principal $G$-bundles. The following is proved (see Propositions 2.6 and 3.2):

Proposition 1.1. A pseudo-real principal $G$-bundle $\left(E_{G}, \rho\right)$ is semistable (respectively, polystable) if and only if $E_{G}$ is semistable (respectively, polystable).

A comment on the definition of (semi)stability is in order. Ramanathan and Behrend defined (semi)stable principal bundles $[3,11]$. It seems to be a common belief that their definitions are equivalent. When the base field is algebraically closed, it is easy to see that the two definitions are equivalent. But here we are working over the base field $\mathbb{R}$. In turns out that in this case their definitions differ. The definition of Behrend works better. (See Section 2.3 for the details.)

Fix a maximal compact subgroup $K \subset G$ such that $\sigma_{G}(K)=K$ (we show that such a subgroup exists). Let

$$
\widehat{K}:=(\mathbb{Z} / 2 \mathbb{Z}) \ltimes K
$$

be the semi-direct product given by the involution $\sigma_{G}$. Fix a point $x \in X$ such that $\sigma(x) \neq x$. Let $\Gamma$ be the space of all homotopy classes of paths on $X$ starting from $x$ and ending in either $x$ or $\sigma(x)$. This set $\Gamma$ has a natural structure of a group. Let $\operatorname{Hom}^{\prime}(\Gamma, \widehat{K})$ be the space of all homomorphisms $\varphi: \Gamma \longrightarrow \widehat{K}$ that fit in the commutative diagram

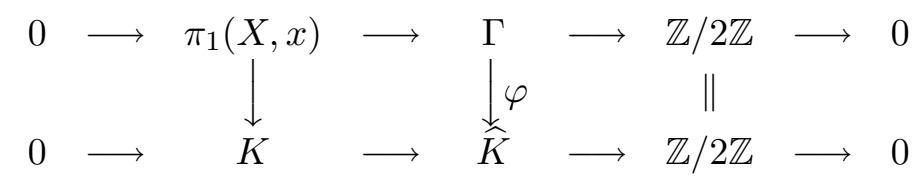

We prove the following theorem (see Theorem 3.4):

Theorem 1.2. Let $G$ be semisimple. Isomorphism classes of polystable real principal $G$-bundles on $X$ are in bijective correspondence with $\operatorname{Hom}^{\prime}(\Gamma, \widehat{K}) / K$. 
Let us assume that the involutions $\sigma$ and $\sigma_{G}$ are such that $\sigma^{*} \bar{E}_{G}$ and $E_{G}$ are of the same topological type. Let $\mathcal{M}_{X}(G)$ be the moduli space of stable principal $G$-bundles on $X$ of the given topological type. The smooth locus of $\mathcal{M}_{X}(G)$ will be denoted by $\mathcal{M}_{X}^{s}(G)$. We have an anti-holomorphic involution

$$
\eta: \mathcal{M}_{X}^{s}(G) \longrightarrow \mathcal{M}_{X}^{s}(G)
$$

defined by $E_{G} \longmapsto \sigma^{*} \bar{E}_{G}$.

We prove the following (see Theorem 4.1):

Theorem 1.3. Assume that genus $(X) \geq 3$. Take any principal $G$-bundle $E_{G} \in \mathcal{M}_{X}^{s}(G)$. Then this $E_{G}$ is fixed by the involution $\eta$ if and only if $E_{G}$ admits a pseudo-real structure.

\section{Principal bundles and semistability over a curve defined over $\mathbb{R}$}

\subsection{Pseudo-real principal bundles}

Let $G$ be a connected complex algebraic group. Let $\bar{G}$ be the complex algebraic group given by $G$ using the automorphism of the base field $\mathbb{C}$ defined by $\lambda \longmapsto \bar{\lambda}$. Let

$$
\sigma_{G}: G \longrightarrow G
$$

be an anti-holomorphic involutive homomorphism, meaning $\sigma_{G} \circ \sigma_{G}=\operatorname{Id}_{G}$. So $\sigma_{G}$ is an algebraic isomorphism of $G$ with $\bar{G}$. Let

$$
G_{\mathbb{R}} \subset G
$$

be the fixed point set for $\sigma_{G}$; it is a real analytic group. The center of $G$ will be denoted by $Z$. The intersection

$$
Z_{\mathbb{R}}:=Z \cap G_{\mathbb{R}}
$$

coincides with the center of $G_{\mathbb{R}}$. Indeed, this follows immediately from the fact that $G_{\mathbb{R}}$ is Zariski dense in $G$. 
Let $X$ be a compact connected Riemann surface equipped with an antiholomorphic involution

$$
\sigma: X \longrightarrow X
$$

Let $E_{G} \longrightarrow X$ be a holomorphic principal $G$-bundle over $X$. Let

$$
\bar{E}_{G}=E_{G}\left(\sigma_{G}\right):=E_{G} \times{ }^{\sigma_{G}} G \longrightarrow X
$$

be the $C^{\infty}$ principal $G$-bundle over $X$ obtained by extending the structure group of $E_{G}$ using the homomorphism $\sigma_{G}$ in (2.1). So $\bar{E}_{G}$ is a holomorphic principal $\bar{G}$-bundle over $X$.

Remark 2.1. The total space of $\bar{E}_{G}$ is canonically identified with the total space of $E_{G}$. This identification sends any $z \in E_{G}$ to the element of $\bar{E}_{G}$ given by $(z, e) \in E_{G} \times G$; recall that the total space of $\bar{E}_{G}$ is the quotient of $E_{G} \times G$ where two elements $\left(z_{1}, g_{1}\right)$ and $\left(z_{2}, g_{2}\right)$ of $E_{G} \times G$ are identified in $\bar{E}_{G}$ if and only if there is an element $h \in G$ such that $z_{2}=z_{1} h$ and $g_{2}=\sigma_{G}\left(h^{-1}\right) g_{1}$. The inverse map $\bar{E}_{G} \longrightarrow E_{G}$ is obtained from the map $E_{G} \times G \longrightarrow E_{G}$ defined by $(z, g) \longmapsto z \sigma_{G}^{-1}(g)$.

Remark 2.2. We note that there is no natural holomorphic structure on the principal $G$-bundle $\bar{E}_{G}$ over $X$ because $\sigma_{G}$ is not holomorphic (although it is a holomorphic principal $\bar{G}$-bundle). On the other hand, since both $\sigma_{G}$ and $\sigma$ (defined in (2.4)) are anti-holomorphic, the pulled back principal $G$-bundle $\sigma^{*} \bar{E}_{G}$ has a natural holomorphic structure given by the holomorphic structure on $E_{G}$. To describe the holomorphic structure on $\sigma^{*} \bar{E}_{G}$, first note that the total space of $\sigma^{*} \bar{E}_{G}$ is naturally identified with the total space of $\bar{E}_{G}$; one can keep the set (for the total space) fixed, and simply modify the projection to $X$ by $\sigma$. In Remark 2.1, we saw that the total space of $\bar{E}_{G}$ is identified with the total space of $E_{G}$; indeed it is the same set, but with the action of $G$ twisted by $\sigma_{G}$. Combining these two identifications, we obtain a natural identification of $\sigma^{*} \bar{E}_{G}$ with $E_{G}$; this identification does not commute with projection to $X$, however, but must be intertwined with $\sigma$, i.e., the identification is a lift of $\sigma$. With this identification, the holomorphic structure on $\sigma^{*} \bar{E}_{G}$ is uniquely determined by the condition that the identification between the total spaces of $\sigma^{*} \bar{E}_{G}$ and $E_{G}$ is anti-holomorphic.

As both $\sigma$ and $\sigma_{G}$ are involutions, the principal $G$-bundles $\sigma^{*} \sigma^{*} E_{G}$ and $\overline{\bar{E}}_{G}$ are identified with $E_{G}$. We also note that $\sigma^{*} \bar{E}_{G}$ is identified with $\bar{\sigma}^{*} E_{G}$. 
For any $z \in Z$ (see $(2.3)$ ), and any holomorphic principal $G$-bundle $F_{G}$, the map $F_{G} \longrightarrow F_{G}$ defined by $y \longmapsto y z$ is a holomorphic isomorphism of principal $G$-bundles. For any $z \in Z \backslash\{e\}$, the holomorphic automorphism of $F_{G}$ given by $z$ is nontrivial. Therefore, we have

$$
Z \subset \operatorname{Aut}\left(F_{G}\right)
$$

where $\operatorname{Aut}\left(F_{G}\right)$ is the group of all holomorphic automorphisms of the principal $G$-bundle $F_{G}$ over the identity map of $X$.

Definition 2.3. A pseudo-real principal $G$-bundle on $X$ is a pair of the form $\left(E_{G}, \rho\right)$, where $E_{G} \longrightarrow X$ is a holomorphic principal $G$-bundle, and

$$
\rho: E_{G} \longrightarrow \sigma^{*} \bar{E}_{G}
$$

is a holomorphic isomorphism of principal $G$-bundles satisfying the condition that there is an element $c \in Z_{\mathbb{R}}$ such that the composition

$$
E_{G} \stackrel{\rho}{\longrightarrow} \sigma^{*} \bar{E}_{G} \stackrel{\sigma^{*} \bar{\rho}}{\longrightarrow} \sigma^{*} \bar{\sigma}^{*} \bar{E}_{G}=\sigma^{*} \sigma^{*} \overline{\bar{E}}_{G}=E_{G}
$$

coincides with the automorphism of $E_{G}$ given by $c$.

Some clarifications on the above definition are in order. The morphism

$$
\bar{\rho}: \bar{E}_{G} \longrightarrow \bar{\sigma}^{*} \bar{E}_{G}
$$

in Definition 2.3 is the one given by $\rho$ using the natural identifications of $E_{G}$ and $\sigma^{*} \bar{E}_{G}$ with $\bar{E}_{G}$ and $\bar{\sigma}^{*} \bar{E}_{G}$ respectively (see Remark 2.1). Since $\sigma^{*} \bar{E}_{G}={\overline{\sigma^{*} E_{G}}}_{G}$, it follows immediately that $\sigma^{*}{\overline{\sigma^{*}}}_{\bar{E}_{G}}=\sigma^{*} \sigma^{*} \overline{\bar{E}}_{G}$. The element $c$ in Definition 2.3 is unique because $Z$ is a subgroup of $\operatorname{Aut}\left(E_{G}\right)$ (see (2.5)).

An isomorphism between two pseudo-real principal $G$-bundles $\left(E_{G}, \rho\right)$ and $\left(F_{G}, \delta\right)$ is a holomorphic isomorphism of principal $G$-bundles

$$
\mu: E_{G} \longrightarrow F_{G}
$$

such that the following diagram commutes:

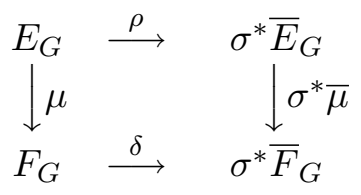


where $\sigma^{*} \bar{\mu}$ is the holomorphic isomorphism of principal $G$-bundles given by $\mu$; the map $\sigma^{*} \bar{\mu}$ coincides with $\mu$ using the identification of the total spaces of $E_{G}$ and $F_{G}$ with $\sigma^{*} \bar{E}_{G}$ and $\sigma^{*} \bar{F}_{G}$ respectively (see Remark 2.2).

Definition 2.4. A real principal $G$-bundle on $X$ is a pseudo-real principal $G$-bundle $\left(E_{G}, \rho\right)$ such that the composition

$$
E_{G} \stackrel{\rho}{\longrightarrow} \sigma^{*} \bar{E}_{G} \stackrel{\sigma^{*} \bar{\rho}}{\longrightarrow} \sigma^{*} \bar{\sigma}^{*} \bar{E}_{G}=\sigma^{*} \sigma^{*} \overline{\bar{E}}_{G}=E_{G}
$$

is the identity automorphism.

Therefore, a pseudo-real principal $G$-bundle $\left(E_{G}, \rho\right)$ as in Definition 2.3 is real if and only if $c=e$.

An alternate way of viewing these structures is as anti-holomorphic lifts

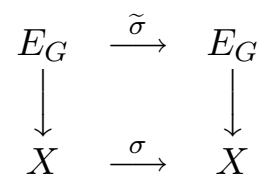

such that $\widetilde{\sigma}(z \cdot g)=\widetilde{\sigma}(z) \cdot \sigma_{G}(g)$ and the composition $\widetilde{\sigma} \circ \widetilde{\sigma}$ is fiber-wise multiplication by $c$; see Remark 2.2 .

Consider $\widetilde{\sigma}$ in (2.6). We can modify the lift $\widetilde{\sigma}$ by the action of an element $a$ of $Z$ as follows: $\widetilde{\sigma}^{\prime}(z):=\widetilde{\sigma}(z) \cdot a$. This then gives the constraint $a \sigma_{G}(a) \in Z_{\mathbb{R}}$, and the element $c$ gets replaced by $a \sigma_{G}(a) c=c a \sigma_{G}(a)$.

In particular, we can take $a$ lying in $Z_{\mathbb{R}}$, and the composition gets altered by $a^{2}$. Therefore, if $c$ lies in $Z_{\mathbb{R}}^{2}:=\left\{z^{2} \mid z \in Z_{\mathbb{R}}\right\}$, or more generally is of the form $\sigma_{G}(a) a$, we can normalize our pseudo-real structure to a real one.

Let $Z_{\mathbb{R}}(2)$ be the group of points of $Z_{\mathbb{R}}$ of order 2 . The natural homomorphism $Z_{\mathbb{R}}(2) \longrightarrow Z_{\mathbb{R}} / Z_{\mathbb{R}}^{2}$ is surjective. So one can assume, as we will from now on, that the element $c$ in Definition 2.3 is of order two. In particular, it lies in any maximal compact subgroup of $G$.

\subsection{Stable and semistable principal bundles}

Henceforth, we assume that the group $G$ is reductive.

Let $\left(E_{G}, \rho\right)$ be a pseudo-real principal $G$-bundle over $X$. Let

$$
\operatorname{Ad}\left(E_{G}\right):=E_{G} \times{ }^{G} G \longrightarrow X
$$

be the group-scheme over $X$ associated with $E_{G}$ for the adjoint action of $G$ on itself. So $\operatorname{Aut}\left(E_{G}\right)$ in (2.5) is the space of all holomorphic sections of 
$\operatorname{Ad}\left(E_{G}\right)$. Consider the $C^{\infty}$ principal $G$-bundle $\bar{E}_{G}$. Since it is, by definition, the extension of structure group of $E_{G}$ by the isomorphism $\sigma_{G}$ in (2.1), the homomorphism $\sigma_{G}$ induces a $C^{\infty}$ isomorphism

$$
\alpha: \operatorname{Ad}\left(E_{G}\right) \longrightarrow \operatorname{Ad}\left(\bar{E}_{G}\right),
$$

where $\operatorname{Ad}\left(\bar{E}_{G}\right)$ is the adjoint bundle for $\bar{E}_{G}$. More precise, both $\operatorname{Ad}\left(E_{G}\right)$ and $\operatorname{Ad}\left(\bar{E}_{G}\right)$ are quotients of $E_{G} \times G$; the map $\alpha$ in (2.7) is the descent of the self-map $\operatorname{Id}_{E_{G}} \times \sigma_{G}$ of $E_{G} \times G$. Note that for each point $x \in X$, the restriction

$$
\alpha(x): \operatorname{Ad}\left(E_{G}\right)_{x} \longrightarrow \operatorname{Ad}\left(\bar{E}_{G}\right)_{x}
$$

is an isomorphism of groups.

The isomorphism $\rho$ produces a holomorphic isomorphism

$$
\operatorname{Ad}\left(E_{G}\right) \longrightarrow \operatorname{Ad}\left(\sigma^{*} \bar{E}_{G}\right)=\sigma^{*} \operatorname{Ad}\left(\bar{E}_{G}\right) .
$$

Combining this with $\alpha$ in (2.7), we obtain an anti-holomorphic automorphism $\widetilde{\rho}$ on $\operatorname{Ad}\left(E_{G}\right)$ over $\sigma$, meaning $\widetilde{\rho}$ fits in the commutative diagram

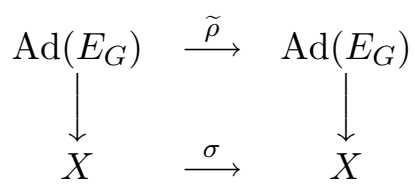

We note that $\widetilde{\rho} \circ \widetilde{\rho}=\operatorname{Id}_{\mathrm{Ad}\left(E_{G}\right)}$ because the adjoint action of $Z_{\mathbb{R}}$ on $G$ is the trivial one.

Let

$$
\operatorname{ad}\left(E_{G}\right):=E_{G} \times{ }^{G} \mathfrak{g} \longrightarrow X
$$

be the bundle of Lie algebras over $X$ associated with $E_{G}$ for the adjoint action of $G$ on $\mathfrak{g}:=\operatorname{Lie}(G)$; it is called the adjoint vector bundle.

A proper parabolic subgroup-scheme of $\operatorname{Ad}\left(E_{G}\right)$ is a Zariski closed analytically locally trivial proper subgroup-scheme $P \subset \operatorname{Ad}\left(E_{G}\right)$ such that $\operatorname{Ad}\left(E_{G}\right) / P$ is compact. For an analytically locally trivial subgroup-scheme $P \subset \operatorname{Ad}\left(E_{G}\right)$, let $\mathfrak{p} \subset \operatorname{ad}\left(E_{G}\right)$ be the bundle of Lie subalgebras corresponding to $P$.

Definition 2.5. A pseudo-real principal $G$-bundle $\left(E_{G}, \rho\right)$ over $X$ is called semistable (respectively, stable) if for every proper parabolic subgroup-scheme 
$P \subset \operatorname{Ad}\left(E_{G}\right)$ such that $\widetilde{\rho}(P) \subset P$, where $\widetilde{\rho}$ is constructed in (2.8),

$$
\operatorname{degree}(\mathfrak{p}) \leq 0 \quad \text { (respectively, degree }(\mathfrak{p})<0)
$$

(the vector bundle $\mathfrak{p}$ is defined above).

The above definition coincides with the one in [3, page 304, Definition 8.1], but it differs from the definition of (semi)stability given in [11]. The definitions of Behrend and Ramanathan are equivalent if the base field is $\mathbb{C}$. The difference between the definitions in [3] and [11] of (semi)stable principal bundles will be explained in Section 2.3.

For holomorphic vector bundles on $X$ (so the base field is $\mathbb{C}$ ), we will adopt Ramanathan's definition of (semi)stability. We reiterate that in this case, this definition is equivalent to the one given in [3].

See [11], [2, page 221, Definition 3.5] for the definition of a polystable principal bundle over a compact Riemann surface.

Proposition 2.6. A pseudo-real principal $G$-bundle $\left(E_{G}, \rho\right)$ over $X$ is semistable (respectively, stable) if the principal $G$-bundle $E_{G}$ is semistable (respectively, stable).

For a semistable pseudo-real principal $G$-bundle $\left(E_{G}, \rho\right)$, the principal $G$-bundle $E_{G}$ is semistable.

If $\left(E_{G}, \rho\right)$ is a stable pseudo-real principal $G$-bundle over $X$, then the principal $G$-bundle $E_{G}$ is polystable.

Proof. Given a parabolic subgroup-scheme $P \subset \operatorname{Ad}\left(E_{G}\right)$, there is a parabolic subgroup $Q \subset G$ and a holomorphic reduction of structure group $E_{Q} \subset E_{G}$ such that the subgroup-scheme $\operatorname{Ad}\left(E_{Q}\right) \subset \operatorname{Ad}\left(E_{G}\right)$ coincides with $P$ (see the proof of Lemma 2.11 in [2]). (The parabolic subgroup-scheme $P$ defines a conjugacy class of parabolic subgroups of $G$, and the reduction of structure group $E_{Q}$ depends only on the choice of a parabolic subgroup $Q$ in this conjugacy class.) Since any parabolic subgroup-scheme is given by a reduction of structure group to a parabolic subgroup, it follows immediately that $\left(E_{G}, \rho\right)$ is semistable (respectively, stable) if the principal $G$-bundle $E_{G}$ is semistable (respectively, stable).

To prove that $E_{G}$ is semistable if $\left(E_{G}, \rho\right)$ is so, assume that $E_{G}$ is not semistable. Then the adjoint vector bundle $\operatorname{ad}\left(E_{G}\right)$ is not semistable $[1$, page 698, Lemma 3]. Using the Harder-Narasimhan filtration of $\operatorname{ad}\left(E_{G}\right)$, we get a parabolic subalgebra bundle $\mathfrak{p}$ of $\operatorname{ad}\left(E_{G}\right)$, which in turn gives a parabolic 
subgroup-scheme

$$
P \subset \operatorname{Ad}\left(E_{G}\right)
$$

[1, page 699, Lemma 4]. We recall from [1] that the Harder-Narasimhan filtration of $\operatorname{ad}\left(E_{G}\right)$ is of the form

$$
0=E_{-\ell-1} \subset E_{-\ell} \subset \cdots \subset E_{-1} \subset E_{0} \subset E_{1} \subset \cdots \subset E_{\ell-1} \subset E_{\ell}=\operatorname{ad}\left(E_{G}\right)
$$

for some positive integer $\ell$. The subbundle $\mathfrak{p}$ mentioned above is $E_{0}$ in (2.10).

Let $\beta: \operatorname{ad}\left(E_{G}\right) \longrightarrow \operatorname{ad}\left(\bar{E}_{G}\right)$ be the $C^{\infty}$ isomorphism given by $\alpha$ in $(2.7)$. Using $\beta$, any $C^{\infty}$ subbundle $V \subset \operatorname{Ad}\left(E_{G}\right)$ produces a subbundle of $\operatorname{ad}\left(\sigma^{*} \bar{E}_{G}\right)=\sigma^{*} \operatorname{ad}\left(\bar{E}_{G}\right)$, which we will denote by $V^{\prime}$. This subbundle $V^{\prime}$ is holomorphic if and only if $V$ is a holomorphic subbundle. (If $h: M \longrightarrow N$ is an anti-holomorphic isomorphism between complex manifolds, then a $C^{\infty}$ submanifold $M^{\prime} \subset M$ is complex analytic if and only if $f\left(M^{\prime}\right)$ is complex analytic.)

For $i \in[-\ell-1, \ell]$, let

$$
E_{i}^{\prime} \subset \operatorname{ad}\left(\sigma^{*} \bar{E}_{G}\right)
$$

be the holomorphic subbundle corresponding to $E_{i}$ in $(2.10)$.

Let

$$
\rho_{\mathrm{ad}}: \operatorname{ad}\left(E_{G}\right) \longrightarrow \operatorname{ad}\left(\sigma^{*} \bar{E}_{G}\right)
$$

be the holomorphic isomorphism induced by $\rho$. Note that $\rho_{\text {ad }}$ is an involution because the central element $c$ in Definition 2.3 acts trivially on $\mathfrak{g}$. Since

$$
\operatorname{rank}\left(E_{i}^{\prime}\right)=\operatorname{rank}\left(E_{i}\right) \text { and degree }\left(E_{i}^{\prime}\right)=\operatorname{degree}\left(E_{i}\right)
$$

and $\operatorname{ad}\left(E_{G}\right)$ is holomorphically isomorphic to $\operatorname{ad}\left(\sigma^{*} \bar{E}_{G}\right)$, we conclude that

$$
0=E_{-\ell-1}^{\prime} \subset E_{-\ell}^{\prime} \subset \cdots \subset E_{-1}^{\prime} \subset E_{0}^{\prime} \subset E_{1}^{\prime} \subset \cdots \subset E_{\ell-1}^{\prime} \subset E_{\ell}^{\prime}=\operatorname{ad}\left(\sigma^{*} \bar{E}_{G}\right)
$$

is the Harder-Narasimhan filtration of $\operatorname{ad}\left(\sigma^{*} \bar{E}_{G}\right)$. Indeed, for any $i \in[-\ell-$ $1, \ell-1]$, the quotient $E_{i+1} / E_{i}$ is the unique subbundle of $\operatorname{ad}\left(E_{G}\right) / E_{i}$ of maximal rank among the subbundles of maximal slope. From this it follows that the above filtration of $\operatorname{ad}\left(\sigma^{*} \bar{E}_{G}\right)$ coincides with its Harder-Narasimhan filtration. 
Since any holomorphic isomorphism between vector bundles preserves their Harder-Narasimhan filtrations, we conclude that

$$
\rho_{\mathrm{ad}}\left(E_{0}\right)=E_{0}^{\prime}
$$

where $\rho_{\text {ad }}$ is constructed in (2.11). This implies that $\widetilde{\rho}(P) \subset P$, where $\widetilde{\rho}$ is constructed in (2.8), and $P$ is the subgroup-scheme in (2.9).

We note that

$$
\operatorname{degree}\left(\operatorname{ad}\left(E_{G}\right)\right)=0
$$

Indeed, any $G$-invariant nondegenerate symmetric bilinear form on $\mathfrak{g}$ produces a nondegenerate symmetric bilinear form on $\operatorname{ad}\left(E_{G}\right)$. This bilinear form on $\operatorname{ad}\left(E_{G}\right)$ identifies $\operatorname{ad}\left(E_{G}\right)$ with its dual $\operatorname{ad}\left(E_{G}\right)^{*}$. In particular, (2.12) holds.

We have

$$
\operatorname{degree}\left(E_{0} / E_{-1}\right)=0
$$

implying that degree $\left(E_{-1}\right)>0$ (see [2, page 216]). Hence in view of $(2.12)$ we conclude that the subbundle $E_{0}=\mathfrak{p} \subset \operatorname{ad}\left(E_{G}\right)$ violates the inequality in Definition 2.5. So $\left(E_{G}, \rho\right)$ is not semistable. This proves the second part of the proposition.

To prove the third part of the proposition, let $\left(E_{G}, \rho\right)$ be a stable pseudoreal principal $G$-bundle over $X$. From the second part of the proposition we know that $E_{G}$ is semistable. Assume that $E_{G}$ is not polystable. Since $E_{G}$ is semistable but not polystable, the adjoint vector bundle $\operatorname{ad}\left(E_{G}\right)$ is semistable but not polystable (see [2, page 214, Proposition 2.10] and [2, page 224, Corollary 3.8]). Let

$$
S \subset \operatorname{ad}\left(E_{G}\right)
$$

be the unique maximal polystable subbundle, which is called socle, of $\operatorname{ad}\left(E_{G}\right)$ (see [7, page 23, Lemma 1.5.5]).

We noted in the proof of the second part of the proposition that any holomorphic subbundle of $\operatorname{ad}\left(E_{G}\right)$ produces a holomorphic subbundle of $\operatorname{ad}\left(\sigma^{*} \bar{E}_{G}\right)$. Let

$$
S^{\prime} \subset \operatorname{ad}\left(\sigma^{*} \bar{E}_{G}\right)
$$

be the holomorphic subbundle corresponding to the socle $S$. 
We will show that

$$
\rho_{\mathrm{ad}}(S)=S^{\prime}
$$

where $\rho_{\text {ad }}$ is constructed in $(2.11)$. To prove this, first note that $\operatorname{ad}\left(\sigma^{*} \bar{E}_{G}\right)$ is semistable but not polystable, because $\rho_{\text {ad }}$ is an isomorphism of it with $\operatorname{ad}\left(E_{G}\right)$. The holomorphic subbundles of $S$ are in bijective correspondence with the holomorphic subbundles of $S^{\prime}$ by the earlier described correspondence between subbundles of $\operatorname{ad}\left(E_{G}\right)$ and subbundles of $\operatorname{ad}\left(\sigma^{*} \bar{E}_{G}\right)$. Since this correspondence preserves both rank and degree, it follows that $S^{\prime}$ is the socle of $\operatorname{ad}\left(\sigma^{*} \bar{E}_{G}\right)$. Now (2.14) follows from the uniqueness of the socle.

Let $Z_{0}$ be the connected component, containing the identity element, of the center $Z$ of $G$. A holomorphic reduction of structure group $E_{Q} \subset E_{G}$ to a parabolic subgroup $Q$ of $G$ is called admissible if for any character $\chi$ of $Q$ trivial on $Z_{0}$, the associated line bundle $E_{Q} \times \chi \mathbb{C}$ over $X$ is of degree zero. A character $\chi$ of $Q$ is called strictly anti-dominant if $\chi$ is trivial on $Z_{0}$, and the associated line bundle $G \times \chi \mathbb{C}$ on $G / Q$ is ample (we are using the fact that the natural projection $G \longrightarrow G / Q$ is a principal $Q$-bundle).

We will recall a construction from [2].

We have the socle filtration of $\operatorname{ad}\left(E_{G}\right)$

$$
S:=S_{0} \subset S_{1} \subset \cdots \subset S_{k-1} \subset S_{k}:=\operatorname{ad}\left(E_{G}\right)
$$

where $S_{i} / S_{i-1}$ is the socle of $\operatorname{ad}\left(E_{G}\right) / S_{i-1}$ for all $i \in[1, k]$. Using this socle filtration, we get a holomorphic reduction of structure group

$$
E_{Q} \subset E_{G}
$$

where

- $Q \subsetneq G$ is maximal among all the proper parabolic subgroups $Q^{\prime}$ of $G$ such that $E_{G}$ has an admissible reduction of structure group

$$
E_{Q^{\prime}}^{\prime} \subset E_{G}
$$

for which the associated principal $L\left(Q^{\prime}\right)$-bundle

$$
E_{L\left(Q^{\prime}\right)}=E_{Q^{\prime}}^{\prime} / R_{u}\left(Q^{\prime}\right)
$$

is polystable, where $L\left(Q^{\prime}\right):=Q^{\prime} / R_{u}\left(Q^{\prime}\right)$ is the Levi quotient of $Q^{\prime}$, and $R_{u}\left(Q^{\prime}\right)$ is the unique maximal normal unipotent subgroup of $Q^{\prime}$ (also called the unipotent radical of $Q^{\prime}$ ), 
- $E_{Q}$ is a holomorphic reduction of structure group of $E_{G}$ to $Q$ such that the associated principal $L(Q)$-bundle is polystable, where $L(Q)$ is the Levi quotient of $Q$.

The pair $\left(Q, E_{Q}\right)$ is unique in the following sense: for any other pair $\left(Q_{1}, E_{Q_{1}}\right)$ satisfying the above conditions, there is some $g \in G$ such that $Q_{1}=g^{-1} Q g$, and $E_{Q_{1}}=E_{Q} g$. (See [2, page 218].)

Note that from the above relations between $\left(Q, E_{Q}\right)$ and $\left(Q_{1}, E_{Q_{1}}\right)$ it follows that the two subgroup-schemes $\operatorname{Ad}\left(E_{Q}\right)$ and $\operatorname{Ad}\left(E_{Q_{1}}\right)$ of $\operatorname{Ad}\left(E_{G}\right)$ coincide.

From (2.14) it can be deduced that $\widetilde{\rho}\left(\operatorname{Ad}\left(E_{Q}\right)\right)=\operatorname{Ad}\left(E_{Q}\right)$, where $\widetilde{\rho}$ is constructed in (2.8). Indeed, this is evident from the construction of $\operatorname{Ad}\left(E_{Q}\right)$ using the socle filtration.

Since the reduction $E_{Q} \subset E_{G}$ is admissible, and the adjoint action of $Z_{0}$ on $\mathfrak{g}$ is trivial, the degree of the line bundle on $X$ associated with the principal $Q$-bundle $E_{Q}$ for the character of $Q$ given by the $Q$-module $\Lambda^{\text {top }} \operatorname{Lie}(Q)$ is zero. In other words, degree $\left(\operatorname{ad}\left(E_{Q}\right)\right)=0$. This contradicts the fact that $\left(E_{G}, \rho\right)$ is a stable pseudo-real principal $G$-bundle. Therefore, we conclude that $E_{G}$ is polystable.

The following is a corollary of Proposition 2.6:

Corollary 2.7. A pseudo-real principal $G$-bundle $\left(E_{G}, \rho\right)$ over $X$ is semistable if and only if the corresponding adjoint real vector bundle $\left(\operatorname{ad}\left(E_{G}\right), \rho_{\text {ad }}\right)$ (defined in (2.11)) is semistable.

Proof. A pseudo-real principal $G$-bundle $\left(E_{G}, \rho\right)$ over $X$ is semistable if and only if $E_{G}$ is semistable (Proposition 2.6), and we know that $E_{G}$ is semistable if and only if $\operatorname{ad}\left(E_{G}\right)$ is semistable [2, page 214, Proposition 2.10]. But $\operatorname{ad}\left(E_{G}\right)$ is semistable if and only if $\left(\operatorname{ad}\left(E_{G}\right), \rho_{\text {ad }}\right)$ is semistable (recall that $\rho_{\text {ad }}$ is an involution).

It may be mentioned that the analog of Corollary 2.7 for stable bundles is not true. In fact, there are stable holomorphic vector bundles such that the corresponding trace zero endomorphism bundle is not stable (see [8, page 2212, Example 2 and Remark 2]).

\subsection{Ramanathan's definition and Behrend's definition}

We will first recall Ramanathan's definition of (semi)stability in our context.

Let $\left(E_{G}, \rho\right)$ be a pseudo-real principal $G$-bundle over $X$. It will be called $r$-semistable (respectively, r-stable) if for any proper parabolic subgroup 
$Q \subset G$ such that $\sigma_{G}(Q)=Q$, and for any holomorphic reduction of structure group of $E_{G}$

$$
E_{Q} \subset E_{G}
$$

to $Q$ with $\rho\left(E_{Q}\right)=E_{Q}$ (see the next sentence for a clarification), and for any strictly anti-dominant character $\chi$ of $Q$, the line bundle $E_{Q} \times \chi \mathbb{C} \longrightarrow X$ is of nonnegative (respectively, positive) degree. (The total space of $\sigma^{*} \bar{E}_{G}$ is identified with that of $E_{G}$, as shown in Remark 2.2; using this identification, $\rho\left(E_{Q}\right)$ is considered as a submanifold of $\left.E_{G} \cdot\right)$

For any given holomorphic reduction of structure group $E_{Q} \subset E_{G}$ to $Q \subset G$, considering the corresponding subgroup-scheme $\operatorname{Ad}\left(E_{Q}\right) \subset E_{G}$ we conclude that $\left(E_{G}, \rho\right)$ is r-semistable (respectively, r-stable) if $\left(E_{G}, \rho\right)$ is semistable (respectively, stable).

We will construct a r-stable real principal $G$-bundle $\left(E_{G}, \rho\right)$ which is not semistable.

Take a pair $(X, \sigma)$ as in $(2.4)$. Take $G=\mathrm{GL}(2, \mathbb{C})$, and let $\sigma_{G}=\sigma_{\mathrm{GL}(2, \mathbb{C})}$ be the anti-holomorphic involution defined by

$$
A \longmapsto\left(\bar{A}^{t}\right)^{-1} \text {. }
$$

The real subgroup is then $\mathrm{U}(2)$, and the real structure corresponds in vector bundle terms to isomorphisms $V \longrightarrow \sigma^{*}\left(\bar{V}^{*}\right)$ (this will be elaborated later).

Let $L \longrightarrow X$ be a holomorphic line bundle with degree $(L)>0$. Define

$$
M:=\left(\sigma^{*} \bar{L}\right)^{*} \text { and } V:=L \oplus M
$$

Both $M$ and $V$ are holomorphic vector bundles. Note that

$$
\sigma^{*} \bar{V}^{*}=\sigma^{*} \bar{L}^{*} \oplus \sigma^{*} \bar{M}^{*}=M \oplus L=L \oplus M
$$

Therefore, the identity map of $L \oplus M$ produces a holomorphic isomorphism

$$
\rho_{0}: V \longrightarrow \sigma^{*} \bar{V}^{*}
$$

The composition

$$
V \stackrel{\rho_{0}}{\longrightarrow} \sigma^{*} \bar{V}^{*} \sigma^{*} \stackrel{\left({\overline{\rho_{0}}}^{*}\right)^{-1}}{\longrightarrow}\left(\sigma^{*}{\overline{\sigma^{*} \bar{V}^{*}}}^{*}=\sigma^{*} \sigma^{*} \overline{\bar{V}}^{* *}=V\right.
$$

is clearly the identity map of $V$.

The vector bundle $V$ defines a principal $\mathrm{GL}(2, \mathbb{C})$-bundle over $X$, which we will denote by $E_{\mathrm{GL}_{2}}$. We recall that $E_{\mathrm{GL}_{2}}$ is the space of all $\mathbb{C}$-linear 
isomorphisms from $\mathbb{C}^{2}$ to the fibers of $V$. Using $\rho_{0}$ in (2.16), we will construct a map from $E_{\mathrm{GL}_{2}}$ to $\sigma^{*} \bar{E}_{\mathrm{GL}_{2}}$.

Take a point $x \in X$, and take any $\psi \in\left(E_{\mathrm{GL}_{2}}\right)_{x}$ in the fiber over $x$. So, $\psi$ is a $\mathbb{C}$-linear isomorphism from $\mathbb{C}^{2}$ to $V_{x}$. Let

$$
\bar{\psi}: \overline{\mathbb{C}}^{2} \longrightarrow \bar{V}_{x}
$$

be the $\mathbb{C}$-linear isomorphism defined by $v \longmapsto \overline{\psi(\bar{v})}$. Now we have the isomorphism

$$
\left(\bar{\psi}^{*}\right)^{-1}:\left(\overline{\mathbb{C}}^{2}\right)^{*} \longrightarrow\left(\bar{V}_{x}\right)^{*}
$$

On the other hand, the isomorphism $\rho_{0}$ in (2.16) produces an isomorphism

$$
\overline{\rho_{0, x}}: \bar{V}_{x} \longrightarrow\left(\overline{\sigma^{*} \bar{V}^{*}}\right)_{x}=\left(V_{\sigma(x)}\right)^{*}
$$

by sending any $w$ to $\overline{\rho_{0, x}(\bar{w})}$, where $\rho_{0, x}$ is the restriction of $\rho_{0}$ to $x$. Consider the corresponding isomorphism

$$
\left({\overline{\rho_{0, x}}}^{*}\right)^{-1}:\left(\bar{V}_{x}\right)^{*} \longrightarrow\left(V_{\sigma(x)}\right)^{* *}=V_{\sigma(x)} .
$$

Let

$$
\left({\overline{\rho_{0, x}}}^{*}\right)^{-1} \circ\left(\bar{\psi}^{*}\right)^{-1}:\left(\overline{\mathbb{C}}^{2}\right)^{*} \longrightarrow V_{\sigma(x)}
$$

be the composition of it with the isomorphism in (2.17).

Consider the standard inner product on $\mathbb{C}^{2}$; the standard basis is an orthonormal one. Note that the $\mathbb{C}$-linear isometries of $\mathbb{C}^{2}$ are the fixed points of the involution $\sigma_{\mathrm{GL}(2, \mathbb{C})}($ see $(2.15))$. This inner product produces a $\mathbb{C}$-linear isomorphism of $\mathbb{C}^{2}$ with $\left(\overline{\mathbb{C}}^{2}\right)^{*}$. Let

$$
\widetilde{\psi}: \mathbb{C}^{2} \longrightarrow V_{\sigma(x)}
$$

be the isomorphism given by the one in (2.18) using this isomorphism of $\mathbb{C}^{2}$ with $\left(\overline{\mathbb{C}}^{2}\right)^{*}$.

Let

$$
\rho_{\mathrm{GL}}: E_{\mathrm{GL}_{2}} \longrightarrow \sigma^{*} \bar{E}_{\mathrm{GL}_{2}}
$$

be the isomorphism that sends any $\psi$ to $\widetilde{\psi}$ constructed in $(2.19)$. Note that the total space of $\bar{E}_{\mathrm{GL}_{2}}$ is identified with that of $E_{\mathrm{GL}_{2}}$ (the maps are constructed in Remark 2.1); using this identification, the isomorphism $\widetilde{\psi}$ in (2.19) is considered as an element of the fiber $\left(\bar{E}_{\mathrm{GL}_{2}}\right)_{\sigma(x)}$. 
It is straightforward to check that the pair $\left(E_{\mathrm{GL}_{2}}, \rho_{\mathrm{GL}}\right)$ constructed above is a real principal $\mathrm{GL}(2, \mathbb{C})$-bundle over $X$.

We will show that $\left(E_{\mathrm{GL}_{2}}, \rho_{\mathrm{GL}}\right)$ is not semistable. For that, note that for any $x \in X$, the fiber $\operatorname{Ad}\left(E_{\mathrm{GL}_{2}}\right)_{x}$ is $\mathrm{GL}\left(V_{x}\right)$ (the space of all $\mathbb{C}$-linear automorphisms of $V_{x}$ ). Let

$$
P \subset \operatorname{Ad}\left(E_{\mathrm{GL}_{2}}\right)
$$

be the subgroup-scheme whose fiber over any $x \in X$ is the space of all linear automorphisms of $V_{x}=L_{x} \oplus M_{x}$ that preserve the line $L_{x}$. It is straightforward to check that $\widetilde{\rho}(P) \subset P$, where $\widetilde{\rho}$ is the diffeomorphism in (2.8). Let $\mathfrak{p}$ be the Lie algebra bundle corresponding to $P$. We have

$$
\mathfrak{p}=\mathcal{E} n d(L) \oplus \mathcal{E} n d(M) \oplus \mathcal{H} \text { om }(M, L) \subset \mathcal{E} n d(V)=\operatorname{ad}\left(E_{\mathrm{GL}_{2}}\right)
$$

Therefore,

$$
\operatorname{degree}(\mathfrak{p})=\operatorname{degree}(\mathcal{H} \text { om }(M, L))=2 \cdot \operatorname{degree}(L)>0
$$

Hence $\left(E_{\mathrm{GL}_{2}}, \rho_{\mathrm{GL}}\right)$ is not semistable.

On the contrary, $\left(E_{\mathrm{GL}_{2}}, \rho_{\mathrm{GL}}\right)$ is r-stable because there is no proper parabolic subgroup of $\mathrm{GL}(2, \mathbb{C})$ that is preserved by the involution $\sigma_{\mathrm{GL}(2, \mathbb{C})}$. Indeed, for any proper parabolic subgroup $Q$ of $\operatorname{GL}(2, \mathbb{C})$, the intersection $Q \bigcap \sigma_{\mathrm{GL}(2, \mathbb{C})}(Q)$ is isomorphic to $\mathbb{C}^{*} \times \mathbb{C}^{*}$.

Lemma 2.8. Let $\left(E_{G}, \rho\right)$ be a pseudo-real principal $G$-bundle over $X$ satisfying the condition that there is a point $y \in E_{G}$ such that $\rho(y)=y z$ for some element $z$ in the center $Z$ of $G$ (using the identification of the total spaces of $\sigma^{*} \bar{E}_{G}$ and $E_{G}$ (see Remark 2.2), the element $\rho(y)$ of $\sigma^{*} \bar{E}_{G}$ is considered as an element of $\left.E_{G}\right)$. Then $\left(E_{G}, \rho\right)$ is r-semistable if and only if the principal $G$-bundle $E_{G}$ is semistable.

Proof. If $E_{G}$ is semistable, then it is obvious that $\left(E_{G}, \rho\right)$ is r-semistable.

To prove the converse, take a point $y \in E_{G}$ such that $\rho(y)=y z$ with $z \in Z$. Let $x \in X$ be the image of $y$. Since $\rho(y)=y z$, it follows that $\sigma(x)=x$. Let

$$
f_{y}: G \longrightarrow \operatorname{Ad}\left(E_{G}\right)_{x}
$$

be the map that sends any $g \in G$ to the image of $(y, g)$ in $\operatorname{Ad}\left(E_{G}\right)_{x}$ (recall that $\operatorname{Ad}\left(E_{G}\right)_{x}$ is a quotient of $\left.\left(E_{G}\right)_{x} \times G\right)$. This map $f_{y}$ is a holomorphic isomorphism of groups. 
Define $f_{\rho(y)}:=f_{y z}: G \longrightarrow \operatorname{Ad}\left(E_{G}\right)_{x}$ by replacing $y$ with $\rho(y)=y z$ in the above construction of $f_{y}$. The two isomorphisms $f_{y}$ and $f_{y z}$ differ by the automorphism of $G$ produced by the adjoint action of $z$. Since $z$ is in the center of $G$, we conclude that

$$
f_{y}=f_{y z}
$$

From (2.20) it follows that

$$
f_{y}\left(\sigma_{G}(g)\right)=\widetilde{\rho}\left(f_{\rho(y)}(g)\right)=\widetilde{\rho}\left(f_{y}(g)\right)
$$

for all $g \in G$, where $\widetilde{\rho}$ and $\sigma_{G}$ are the maps defined in (2.8) and (2.1) respectively; note that since $\sigma(x)=x$, the map $\widetilde{\rho}$ sends $\operatorname{Ad}\left(E_{G}\right)_{x}$ to itself.

Assume that $E_{G}$ is not semistable. Let $P \subset \operatorname{Ad}\left(E_{G}\right)$ be the parabolic subgroup-scheme in (2.9) constructed from the Harder-Narasimhan filtration of the vector bundle $\operatorname{ad}\left(E_{G}\right)$. Let

$$
Q:=f_{y}^{-1}\left(P_{x}\right) \subset G
$$

be the parabolic subgroup, where $f_{y}$ is the isomorphism constructed above. Since $\widetilde{\rho}(P)=P$, from $(2.21)$ we conclude that

$$
\sigma_{G}(Q)=Q
$$

Let $E_{Q} \subset E_{G}$ be the holomorphic reduction of structure group of $E_{G}$ to $Q$ constructed using the pair $(P, Q)$ (see the proof of Lemma 2.11 in [2] for the construction of $\left.E_{Q}\right)$. Since $\widetilde{\rho}(P)=P$, from (2.22) it follows immediately that $\rho\left(E_{Q}\right)=E_{Q}$. Therefore, the reduction $E_{Q} \subset E_{G}$ establishes that $\left(E_{G}, \rho\right)$ is not r-semistable.

Proposition 2.6 and Lemma 2.8 together give the following corollary:

Corollary 2.9. Let $\left(E_{G}, \rho\right)$ be a pseudo-real principal G-bundle on $X$ satisfying the condition that there is a point $y \in E_{G}$ such that $\rho(y)=y z$ for some $z \in Z$. Then $\left(E_{G}, \rho\right)$ is semistable if and only if it is r-semistable.

Remark 2.10. The assumption in Lemma 2.8 and Corollary 2.9 that $\rho(y)=y z$ implies that the image of $y$ in $X$ is fixed by the involution $\sigma$. Hence this assumption fails if $\sigma$ does not have a fixed point. 


\section{Polystable pseudo-real principal bundles and representations of the fundamental group}

\subsection{Polystable pseudo-real principal bundles}

As before, $G$ is a connected complex reductive group.

Let $E_{G}$ be a holomorphic principal $G$-bundle over $X$. Let $P \subset \operatorname{Ad}\left(E_{G}\right)$ be a proper parabolic subgroup-scheme. For each point $x \in X$, the unipotent radical of the fiber $P_{x}$ will be denoted by $R_{u}(P)_{x}$; it is the unique maximal normal unipotent subgroup. We have an analytically locally trivial subgroupscheme

$$
R_{u}(P) \subset P
$$

whose fiber over any $x \in X$ is $R_{u}(P)_{x}$. The quotient $P / R_{u}(P)$ is a groupscheme over $X$.

A Levi subgroup-scheme of $P$ is an analytically locally trivial subgroupscheme $L(P) \subset P$ such that the composition

$$
L(P) \hookrightarrow P \longrightarrow P / R_{u}(P)
$$

is an isomorphism. It should be emphasized that a Levi subgroup-scheme does not exist in general. In vector bundle terms, the existence of a Levi subgroup-scheme corresponds to some extension classes being trivial.

Definition 3.1. A semistable pseudo-real principal $G$-bundle $\left(E_{G}, \rho\right)$ over $X$ is called polystable if either $\left(E_{G}, \rho\right)$ is stable, or there is a proper parabolic subgroup-scheme $P \subset \operatorname{Ad}\left(E_{G}\right)$, and a Levi subgroup-scheme $L(P) \subset P$, such that the following conditions hold:

1. $\widetilde{\rho}(P) \subset P$ and $\widetilde{\rho}(L(P)) \subset L(P)$, where $\widetilde{\rho}$ is constructed in (2.8), and

2. for any proper parabolic subgroup-scheme $P^{\prime} \subset L(P)$ with $\widetilde{\rho}\left(P^{\prime}\right) \subset P^{\prime}$, we have

$$
\operatorname{degree}\left(\mathfrak{p}^{\prime}\right)<0,
$$

where $\mathfrak{p}^{\prime}$ is the bundle of Lie algebras corresponding to $P^{\prime}$.

It should be clarified that in the above definition, $P^{\prime}$ is not a parabolic subgroup-scheme of $\operatorname{Ad}\left(E_{G}\right)$. The condition that $P^{\prime}$ is a parabolic subgroupscheme of $L(P)$ implies that the quotient $L(P) / P^{\prime}$ is compact.

Proposition 3.2. A pseudo-real principal $G$-bundle $\left(E_{G}, \rho\right)$ is polystable if and only if the principal $G$-bundle $E_{G}$ is polystable. 
Proof. First assume that the pseudo-real principal $G$-bundle $\left(E_{G}, \rho\right)$ is polystable. We will show that $E_{G}$ is polystable.

We begin by constructing a reduction of $E_{G}$ to a suitable parabolic subgroup $Q$. Fix a point $x_{0} \in X$, and also fix a point $z_{0} \in\left(E_{G}\right)_{x_{0}}$ in the fiber over $x_{0}$. Let

$$
\phi: G \longrightarrow \operatorname{Ad}\left(E_{G}\right)_{x_{0}}
$$

be the map that sends any $g \in G$ to the image of $(z, g)$ in $\operatorname{Ad}\left(E_{G}\right)_{x_{0}}$; recall that $\operatorname{Ad}\left(E_{G}\right)_{x_{0}}$ is a quotient of $\left(E_{G}\right)_{x_{0}} \times G$. This map $\phi$ is a holomorphic isomorphism of groups.

If $\left(E_{G}, \rho\right)$ is stable, then $E_{G}$ is polystable by Proposition 2.6. Assume that $\left(E_{G}, \rho\right)$ is not stable. Take $P$ and $L(P)$ as in Definition 3.1. Let

$$
Q:=\phi^{-1}\left(P_{x_{0}}\right) \subset G
$$

be the parabolic subgroup. The conjugacy class of the subgroup $Q$ is independent of the choices of $x_{0}$ and $z_{0}$ (meaning subgroups are conjugate by some element of $G$ ). We will first show that $E_{G}$ admits a natural holomorphic reduction of structure group to $Q$.

For any $x \in X$, let

$$
\left(E_{Q}\right)_{x} \in\left(E_{G}\right)_{x}
$$

be the submanifold consisting of all points $z$ such that for all $q \in Q$, the image of $(z, q) \in\left(E_{G}\right)_{x} \times Q$ in $\operatorname{Ad}\left(E_{G}\right)_{x}$ lies in the subgroup $P_{x}$ (recall that $\operatorname{Ad}\left(E_{G}\right)_{x}$ is a quotient of $\left.\left(E_{G}\right)_{x} \times Q\right)$. These $\left(E_{Q}\right)_{x}, x \in X$, together form a holomorphic sub-fiber bundle $E_{Q} \subset E_{G}$. In fact, $E_{Q}$ is a holomorphic reduction of structure group of the principal $G$-bundle $E_{G}$ to the subgroup $Q$. This follows from the fact that the normalizer of $Q$ in $G$ coincides with $Q$. It should be mentioned that $\rho\left(E_{Q}\right)$ need not coincide with $E_{Q}$ (we consider $\rho\left(E_{Q}\right)$ as a submanifold of the total space of $E_{G}$ using the identification of the total spaces of $E_{G}$ and $\sigma^{*} \bar{E}_{G}$ described in Remark 2.2).

Consider $L(P) \subset P$ as in Definition 3.1. Define

$$
L(Q):=\phi^{-1}\left(L(P)_{x_{0}}\right) \subset Q
$$

where $\phi$ is constructed in (3.1). It is a Levi subgroup of $Q$, meaning $L(Q)$ is a connected reductive subgroup of $Q$ such that the composition

$$
L(Q) \hookrightarrow Q \longrightarrow Q / R_{u}(Q)
$$


is an isomorphism, where $R_{u}(Q)$ is the unipotent radical of $Q$. Any two Levi subgroups of $Q$ are conjugate by some element of $Q$. We will show that $L(P)$ produces a holomorphic reduction of structure group of the principal $Q$-bundle $E_{Q}$ to the subgroup $L(Q) \subset Q$ defined in (3.2).

Let $Z(L(Q)) \subset L(Q)$ be the connected component of the center of $L(Q)$ containing the identity element. It is a product of copies of $\mathbb{C}^{*}$ because $L(Q)$ is reductive. Let

$$
Z(L(P)) \subset L(P)
$$

be the fiber-wise connected component of the center containing the identity element, meaning the fiber $Z(L(P))_{x}$ for any $x \in X$ is the connected component, containing the identity element, of the center of $L(P)_{x}$. So $Z(L(P))_{x}$ is isomorphic to $Z(L(Q))$.

We recall that for any $x \in X$, the fiber $\operatorname{Ad}\left(E_{Q}\right)_{x}$ is the group of all $Q$-equivariant self-maps of $\left(E_{Q}\right)_{x}$. Let

$$
\mathcal{S} \subset E_{Q}
$$

be the subset consisting of all $(x, z), x \in X$ and $z \in\left(E_{Q}\right)_{x}$, such that

$$
\phi(t)(z)=z t
$$

for all $t \in Z(L(Q))$, where $\phi$ is constructed in (3.1). This $\mathcal{S}$ is a holomorphic reduction of structure group of the principal $Q$-bundle $E_{Q}$ to the subgroup $L(Q)$. It should be emphasized that $\rho(\mathcal{S})$ need not coincide with $\mathcal{S}$. Let

$$
E_{L(Q)} \subset E_{Q}
$$

be the principal $L(Q)$-bundle defined by $\mathcal{S}$ in (3.3). We note that the subgroup-scheme $\operatorname{Ad}\left(E_{L(Q)}\right) \subset \operatorname{Ad}\left(E_{Q}\right)$ is identified with $L(P)$. Indeed, this follows immediately from the above construction of $\mathcal{S}$.

We recall from Definition 3.1 that for any proper parabolic subgroupscheme $P^{\prime} \subset L(P)$ with $\widetilde{\rho}\left(P^{\prime}\right) \subset P^{\prime}$,

$$
\operatorname{degree}\left(\mathfrak{p}^{\prime}\right)<0
$$

where $\mathfrak{p}^{\prime}$ is the bundle of Lie algebras corresponding to $P^{\prime}$. In the proof of Proposition 2.6, we saw that the vector bundle $\operatorname{ad}\left(E_{G}\right)$ is polystable if $\left(E_{G}, \rho\right)$ is stable. Repeating verbatim this argument for $E_{L(Q)}$ (defined in $(3.4)$ ), and using (3.5), we conclude that the adjoint vector bundle $\operatorname{ad}\left(E_{L(Q)}\right)$ is polystable. Note that in the proof that $\operatorname{ad}\left(E_{G}\right)$ is polystable if $\left(E_{G}, \rho\right)$ is stable, the involution $\rho$ is not used; only $\widetilde{\rho}$ is used. 
We also note that $E_{L(Q)}$ is polystable because $\operatorname{ad}\left(E_{L(Q)}\right)$ is polystable $[2$, page 224, Corollary 3.8].

Consider the adjoint action of $Z(L(Q))$ on $\mathfrak{g}:=\operatorname{Lie}(G)$. Let

$$
\mathfrak{g}=\bigoplus_{i=1}^{n} V_{i}
$$

be the isotypical decomposition of the $Z(L(Q))$-module $\mathfrak{g}$. Note that each subspace $V_{i} \subset \mathfrak{g}$ is a preserved by the adjoint action of $L(Q)$. For any $i \in$ $[1, n]$, let

$$
E_{V_{i}}:=E_{L(Q)} \times{ }^{L(Q)} V_{i} \longrightarrow X
$$

be the holomorphic vector bundle associated with the principal $L(Q)$-bundle $E_{L(Q)}$ (constructed in $(3.4)$ ) for the $L(Q)$-module $V_{i}$. Since $E_{L(Q)}$ is polystable, the vector bundle $E_{V_{i}}$ is polystable [10, page 285, Theorem 3.18] (this theorem of [10] applies because $Z(L(Q))$ acts on $V_{i}$ through a character).

Since $\left(E_{G}, \rho\right)$ is semistable (see Definition 3.1), from the second part of Proposition 2.6 we know that $E_{G}$ is semistable. Hence the adjoint vector bundle $\operatorname{ad}\left(E_{G}\right)$ is semistable [2, page 214, Proposition 2.10]. From (3.6) we have a decomposition

$$
\operatorname{ad}\left(E_{G}\right)=\bigoplus_{i=1}^{n} E_{V_{i}}
$$

Since $\operatorname{ad}\left(E_{G}\right)$ is semistable, and each $E_{V_{i}}$ is polystable, we conclude that $\operatorname{ad}\left(E_{G}\right)$ is polystable. Hence $E_{G}$ is polystable [2, page 224, Corollary 3.8].

To prove the converse, assume that the principal $G$-bundle $E_{G}$ is polystable. We will prove that $\left(E_{G}, \rho\right)$ is polystable.

The adjoint vector bundle $\operatorname{ad}\left(E_{G}\right)$ is polystable because $E_{G}$ is polystable [2, page 224, Corollary 3.8]. Therefore, $\operatorname{ad}\left(E_{G}\right)$ is semistable. Hence for every proper parabolic subgroup-scheme $P \subset \operatorname{Ad}\left(E_{G}\right)$ such that $\widetilde{\rho}(P) \subset P$, where $\widetilde{\rho}$ is constructed in $(2.8)$, we have

$$
\operatorname{degree}(\mathfrak{p}) \leq 0
$$

( $\mathfrak{p}$ is the bundle of Lie algebras associated with $P$ ). If $\operatorname{degree}(\mathfrak{p})<0$ for every such $P$, then $\left(E_{G}, \rho\right)$ is stable (see Definition 2.5), in particular, it is polystable in that case. 
Assume that

$$
\operatorname{degree}(\mathfrak{p})=0
$$

for a proper parabolic subgroup-scheme $P \subset \operatorname{Ad}\left(E_{G}\right)$ with $\widetilde{\rho}(P) \subset P$. We also assume that the rank of the vector bundle $\mathfrak{p}$ is smallest among all bundles of Lie algebras $\mathfrak{p}^{\prime \prime}$ satisfying the conditions that $\operatorname{degree}\left(\mathfrak{p}^{\prime \prime}\right)=0$ and $\mathfrak{p}^{\prime \prime}$ corresponds to a $\widetilde{\sigma}$-invariant proper parabolic subgroup-scheme of $\operatorname{Ad}\left(E_{G}\right)$.

Since $E_{G}$ is polystable, it has an Einstein-Hermitian connection [11], [2, page 208, Theorem 0.1]. Let $\nabla$ be the connection on $\operatorname{ad}\left(E_{G}\right)$ induced by an Einstein-Hermitian connection on $E_{G}$. We need to choose a Kähler form on $X$ and a maximal compact subgroup of $G$ in order to define an EinsteinHermitian connection on $E_{G}$. But the induced connection on $\operatorname{ad}\left(E_{G}\right)$ is independent of the choices of Kähler form on $X$ and maximal compact subgroup of $G$. The connection $\nabla$ on $\operatorname{ad}\left(E_{G}\right)$ is flat unitary. Note that degree $\left(\operatorname{ad}\left(E_{G}\right)\right)=0($ see $(2.12))$.

From (3.7) it follows immediately that the second fundamental form of the subbundle $\mathfrak{p} \subset \operatorname{ad}\left(E_{G}\right)$ for the connection $\nabla$ vanishes identically [9, page 139]. Therefore, the connection $\nabla$ on $\operatorname{ad}\left(E_{G}\right)$ preserves the subbundle $\mathfrak{p}$.

Fix a maximal compact subgroup

$$
K \subset G
$$

such that $\sigma_{G}(K)=K$, where $\sigma_{G}$ is the involution in (2.1). To see that such a subgroup exists, let

$$
\widehat{G}:=(\mathbb{Z} / 2 \mathbb{Z}) \ltimes G
$$

be the semi-direct product for the involution $\sigma_{G}$ in (2.1). Let $\widehat{K} \subset \widehat{G}$ be a maximal compact subgroup. Then, the intersection $\widehat{K} \cap G$ is a maximal compact subgroup of $G$ which is preserved by $\sigma_{G}$.

Fix a Kähler form $\omega$ on $X$ such that $\sigma^{*} \omega=-\omega$, where $\sigma$ is the involution in (2.4); such a Kähler form is constructed by averaging, with respect to $\sigma$, a Hermitian structure on $T X$. Let

$$
E_{K} \subset E_{G}
$$

be a $C^{\infty}$ reduction of structure group of the principal $G$-bundle $E_{G}$ giving the Einstein-Hermitian connection on $E_{G}$.

Fix an inner product $h$ on $\mathfrak{g}$ such that $h$ is preserved by the adjoint action of $K$ on $\mathfrak{g}$ (we note that such an inner product exists because $K$ is compact). Since $\operatorname{ad}\left(E_{G}\right)$ is identified with the vector bundle associated with 
the principal $K$-bundle $E_{K}$ for the adjoint action of $K$ on $\mathfrak{g}$, from the fact that $h$ is preserved by the adjoint action of $K$ it follows immediately that the inner product $h$ on $\mathfrak{g}$ produces a Hermitian structure on $\operatorname{ad}\left(E_{G}\right)$. This Hermitian structure on $\operatorname{ad}\left(E_{G}\right)$ will be denoted by $\widehat{h}$.

Since the connection $\nabla$ on $\operatorname{ad}\left(E_{G}\right)$ is induced by the Einstein-Hermitian connection on $E_{G}$ given by $E_{K}$, it follows immediately that $\nabla$ coincides with the Chern connection on $\operatorname{ad}\left(E_{G}\right)$ associated with the Hermitian structure $\widehat{h}$ on it.

Let

$$
R_{n}(\mathfrak{p}) \subset \mathfrak{p}
$$

be the subbundle defined by the fiber-wise nilpotent radicals; in other words, for each point $x \in X$, the fiber $R_{n}(\mathfrak{p})_{x}$ is the nilpotent radical of the parabolic subalgebra $\mathfrak{p}_{x}$. We note that $R_{n}(\mathfrak{p})$ is a holomorphic subbundle because the section of $\mathcal{H o m}\left(\bigwedge^{2} \mathfrak{p}^{*}, \mathfrak{p}\right)$ defined by the Lie algebra structure on the fibers of $\mathfrak{p}$ is holomorphic. Let

$$
L(\mathfrak{p}):=R_{n}(\mathfrak{p})^{\perp} \bigcap \mathfrak{p} \subset \mathfrak{p}
$$

be the orthogonal complement, with respect to the Hermitian structure $\widehat{h}$ constructed above, of $L(\mathfrak{p})$ inside $\mathfrak{p}$. We will show that this $C^{\infty}$ subbundle $L(\mathfrak{p})$ is preserved by the connection $\nabla$.

For each point $x \in X$, the fiber $L(\mathfrak{p})_{x}$ is closed under the Lie bracket operation on $\mathfrak{p}_{x}$; in fact, $L(\mathfrak{p})_{x}$ is a Levi subalgebra of $\mathfrak{p}_{x}$, meaning it is a maximal reductive subalgebra and the composition

$$
L(\mathfrak{p})_{x} \hookrightarrow \mathfrak{p}_{x} \longrightarrow \mathfrak{p}_{x} / R_{n}(\mathfrak{p})_{x}
$$

is an isomorphism. All these follow from the fact that the inner product $h$ on $\mathfrak{g}$ is preserved by the adjoint action of $K$. Any $G$-invariant nondegenerate symmetric bilinear form on $\mathfrak{g}$ produces a holomorphic nondegenerate symmetric bilinear form on $\operatorname{ad}\left(E_{G}\right)$; the restriction to $L(\mathfrak{p})$ of this holomorphic bilinear form on $\operatorname{ad}\left(E_{G}\right)$ is nondegenerate. Therefore, the $C^{\infty}$ vector bundle $L(\mathfrak{p})$ is isomorphic to its dual $L(\mathfrak{p})^{*}$.

We have degree $(L(\mathfrak{p}))=0$ because $L(\mathfrak{p})^{*}$ is isomorphic to $L(\mathfrak{p})$. Therefore, from (3.7) we conclude that

$$
\operatorname{degree}\left(R_{n}(\mathfrak{p})\right)=0
$$

(recall that $\left.\mathfrak{p}=R_{n}(\mathfrak{p}) \bigoplus L(\mathfrak{p})\right)$. This implies that the unitary flat connection $\nabla$ preserves the holomorphic subbundle $R_{n}(\mathfrak{p})$ of $\operatorname{ad}\left(E_{G}\right)$ [9, page 139]. 
We already proved that $\mathfrak{p}$ is preserved by $\nabla$. Consequently, the orthogonal complement of $R_{n}(\mathfrak{p})$ in $\mathfrak{p}$, namely $L(\mathfrak{p})$, is preserved by $\nabla$. In particular, $L(\mathfrak{p})$ is a holomorphic subbundle of $\mathfrak{p}$.

Using the exponential map, the bundle of subalgebras $L(\mathfrak{p}) \subset \mathfrak{p}$ produces a Levi subgroup-scheme $L(P) \subset P$. More precisely, $L(P)$ is the unique subgroup-scheme of $P$ such that the corresponding bundle of Lie subalgebras coincides with $L(\mathfrak{p})$.

Since $\sigma_{G}(K)=K$, and $\sigma^{*} \omega=-\omega$, it follows that $\widetilde{\rho}(L(P))=L(P)$. Since $\mathfrak{p}$ is of smallest rank among all Lie algebra bundles of degree zero associated with proper parabolic subgroup-schemes of $\operatorname{Ad}\left(E_{G}\right)$ preserved by $\widetilde{\rho}$, we conclude that for any proper parabolic subgroup-scheme $P^{\prime} \subset L(P)$ with $\widetilde{\rho}\left(P^{\prime}\right) \subset P^{\prime}$, the inequality

$$
\operatorname{degree}\left(\mathfrak{p}^{\prime}\right)<0
$$

holds, where $\mathfrak{p}^{\prime}$ is the bundle of Lie algebras corresponding to $P^{\prime}$. Hence $\left(E_{G}, \rho\right)$ is polystable. This completes the proof of the proposition.

The following is an analog of Corollary 2.7.

Corollary 3.3. A pseudo-real principal $G$-bundle $\left(E_{G}, \rho\right)$ over $X$ is polystable if and only if the corresponding adjoint real vector bundle $\left(\operatorname{ad}\left(E_{G}\right), \rho_{\text {ad }}\right)$ is polystable.

Proof. A pseudo-real principal $G$-bundle $\left(E_{G}, \rho\right)$ is polystable if and only if the principal $G$-bundle $E_{G}$ is polystable (Proposition 3.2), and $E_{G}$ is polystable if and only if $\operatorname{ad}\left(E_{G}\right)$ is polystable [2, page 224, Corollary 3.8]. But $\operatorname{ad}\left(E_{G}\right)$ is polystable if and only if $\left(\operatorname{ad}\left(E_{G}\right), \rho_{\text {ad }}\right)$ is polystable (Proposition 3.2).

\subsection{Homomorphisms from fundamental group to a maximal compact subgroup}

In this subsection we assume that the group $G$ is semisimple.

As in (3.8), fix a maximal compact subgroup

$$
K \subset G
$$

such that $\sigma_{G}(K)=K$. Let

$$
K \rtimes(\mathbb{Z} / 2 \mathbb{Z})
$$


be the semi-direct product for the involution $\left.\sigma_{G}\right|_{K}$. The set $\widehat{K}$ is $K \times(\mathbb{Z} / 2 \mathbb{Z})$; the group structure is defined using $\left.\sigma_{G}\right|_{K}$.

Fix a point $x \in X$ such that $\sigma(x) \neq x$. Let $\Gamma_{1}$ denote the homotopy classes of paths on $X$ from $x$ to $\sigma(x)$. We have a group structure on

$$
\Gamma:=\pi_{1}(X, x) \cup \Gamma_{1}
$$

defined as follows: for $\gamma_{1}, \gamma_{2} \in \Gamma$,

- if $\gamma_{2} \in \pi_{1}(X, x)$, then $\gamma_{2} \gamma_{1}$ is simply the composition of paths $\gamma_{1} \circ \gamma_{2}$, and

- if $\gamma_{2} \in \Gamma_{1}$, then $\gamma_{2} \gamma_{1}$ is the composition $\sigma\left(\gamma_{1}\right) \circ \gamma_{2}$ of paths.

(See $[6]$ for more details.)

Composition of paths will be denoted by "o".

The group $\widehat{K}$ in (3.10) and the group $\Gamma$ defined above fit in the exact sequences

$$
0 \longrightarrow K \longrightarrow \widehat{K} \longrightarrow \mathbb{Z} / 2 \mathbb{Z} \longrightarrow 0
$$

and

$$
0 \longrightarrow \pi_{1}(X, x) \longrightarrow \Gamma \longrightarrow \mathbb{Z} / 2 \mathbb{Z} \longrightarrow 0 .
$$

Let $\operatorname{Hom}(\Gamma, \widehat{K})$ be the space of all homomorphisms from $\Gamma$ to the group $\widehat{K}$. Let

$$
\operatorname{Hom}^{\prime}(\Gamma, \widehat{K}) \subset \operatorname{Hom}(\Gamma, \widehat{K})
$$

be the subset consisting of all homomorphisms $\varphi$ such that we have commutative diagram

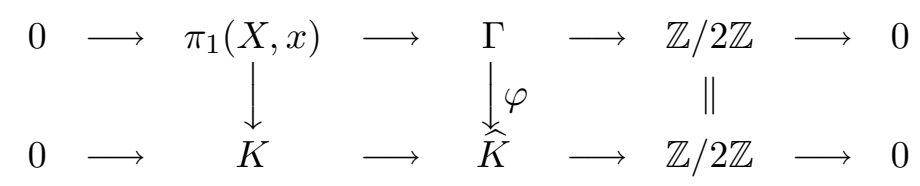

The normal subgroup $K$ of $\widehat{K}$ has a conjugation action on $\operatorname{Hom}^{\prime}(\Gamma, \widehat{K})$.

Theorem 3.4. Let $G$ be a connected semisimple complex affine algebraic group. Isomorphism classes of polystable real principal $G$-bundles on $X$ (see Definition 2.4) are in bijective correspondence with the quotient $\operatorname{Hom}^{\prime}(\Gamma, \widehat{K}) / K$. 
Proof. Let $\left(E_{G}, \rho\right)$ be a polystable real principal $G$-bundle on $X$. So $E_{G}$ is polystable by Proposition 3.2. Therefore, $E_{G}$ has a flat connection given by a $C^{\infty}$ reduction of structure group

$$
E_{K} \subset E_{G},
$$

where $K$ is the subgroup in (3.10); see [11, page 146, Theorem 7.1], [2, page 208, Theorem 0.1]. It is possible to choose $E_{K}$ such that $\rho\left(E_{K}\right)=E_{K}$ (see [5, Proposition 3.7]). We fix a reduction $E_{K}$ such that $\rho\left(E_{K}\right)=E_{K}$. Let $\nabla$ be the flat connection on $E_{K}$.

Fix a point

$$
z_{0} \in\left(E_{K}\right)_{x}
$$

Taking parallel translations of $z_{0}$, for the flat connection $\nabla$, along loops in $X$ based at $x$ we get a homomorphism

$$
\varphi^{\prime}: \pi_{1}(X, x) \longrightarrow K \text {. }
$$

For any $\gamma \in \Gamma_{1}$ (see (3.11)), consider the parallel translation of the element $z_{0}$ in (3.12) along $\gamma$. Let $z_{0}^{\prime} \in\left(E_{K}\right)_{\sigma(x)}$ be the element obtained by this parallel transport. Using the identification of the total space of $E_{G}$ with the total space of $\sigma^{*} \bar{E}_{G}$ (see Remark 2.2), the element $z_{0}^{\prime}$ gives an element $z_{0}^{\prime \prime} \in\left(\sigma^{*} \bar{E}_{G}\right)_{x}$. Therefore,

$$
\rho_{x}^{-1}\left(z_{0}^{\prime \prime}\right) \in\left(E_{G}\right)_{x},
$$

where $\rho_{x}=\left.\rho\right|_{\left(E_{G}\right)_{x}}$.

It can be shown that $\rho_{x}^{-1}\left(z_{0}^{\prime \prime}\right)$ lies in the submanifold $\left(E_{K}\right)_{x} \subset\left(E_{G}\right)_{x}$. To prove this, first observe that $\rho\left(E_{K}\right) \subset E_{G}$ is a reduction of structure group of $E_{G}$ to $\sigma_{G}(K)$ giving a Einstein-Hermitian connection on $E_{G}$ (we have again identified $E_{G}$ with $\sigma^{*} \bar{E}_{G}$ using Remark 2.2). Now, $\rho_{G}(K)=K$, and $\rho$ has the property that it takes the reduction $E_{K}$ of $E_{G}$ to itself (by the choice of $\left.E_{K}\right)$. Hence $\rho_{x}^{-1}\left(z_{0}^{\prime \prime}\right) \in\left(E_{K}\right)_{x}$.

Let

$$
\gamma^{\prime} \in K
$$

be the unique element such that $z_{0} \sigma_{G}\left(\gamma^{\prime}\right)^{-1}=\rho_{x}^{-1}\left(z_{0}^{\prime \prime}\right)$. 
Let

$$
\varphi^{\prime \prime}: \Gamma_{1} \longrightarrow K
$$

be the map defined by $\gamma \longmapsto \gamma^{\prime}$, where $\gamma^{\prime}$ is constructed above from $\gamma$. Let

$$
\varphi: \Gamma \longrightarrow \widehat{K}
$$

be the map defined as follows: $\left.\varphi\right|_{\pi_{1}(X, x)}=\varphi^{\prime}$, where $\varphi^{\prime}$ is constructed in (3.13), and

$$
\varphi(\gamma)=\left(\varphi^{\prime \prime}(\gamma), 1\right) \in K \times(\mathbb{Z} / 2 \mathbb{Z})=\widehat{K}
$$

where $\varphi^{\prime \prime}$ is constructed in (3.14), and $1 \in \mathbb{Z} / 2 \mathbb{Z}$ is the nontrivial element (recall that the natural identification of $\widehat{K}$ with $K \times(\mathbb{Z} / 2 \mathbb{Z})$ is only settheoretic). It is easy to see that

$$
\varphi \in \operatorname{Hom}^{\prime}(\Gamma, \widehat{K})
$$

Conversely, given any

$$
\varphi \in \operatorname{Hom}^{\prime}(\Gamma, \widehat{K})
$$

we will describe a construction of a polystable real principal $G$-bundle on $X$.

Consider the restriction of $\varphi$ to $\pi_{1}(X, x)$. It produces a flat principal $K$-bundle $E_{K}$ on $X$ together with a $K$-equivariant isomorphism

$$
\beta: K \longrightarrow\left(E_{K}\right)_{x}
$$

for the right-translation action of $K$ on itself. Define

$$
z_{0}:=\beta(e) \in\left(E_{K}\right)_{x}
$$

where $e \in K$ is the identity element. The flat connection on $E_{K}$ will be denoted by $\nabla$.

Let $E_{G}:=E_{K} \times{ }^{K} G$ be the principal $G$-bundle obtained by extending the structure group of $E_{K}$ using the inclusion map of $K$ in $G$. The connection $\nabla$ defines a holomorphic structure on $E_{G}$. This holomorphic principal $G$-bundle is polystable because $\nabla$ is flat. 
We will construct an isomorphism of $\left(E_{G}\right)_{x}$ with $\left(E_{G}\right)_{\sigma(x)}$. Take any element

$$
z_{0} g \in\left(E_{G}\right)_{x}
$$

where $g \in G$, and $z_{0}$ is the element in (3.17). Take an element $\gamma \in \Gamma_{1}$ (see $(3.11))$. Let

$$
T_{\gamma}:\left(E_{G}\right)_{x} \longrightarrow\left(E_{G}\right)_{\sigma(x)}
$$

be the parallel translation along $\gamma$. Let

$$
\rho_{x, \gamma}^{\prime}:\left(E_{G}\right)_{x} \longrightarrow\left(E_{G}\right)_{\sigma(x)}
$$

be the map defined by $z_{0} g \longmapsto T_{\gamma}\left(z_{0}\right) \varphi(\gamma) \sigma_{G}(g)$, where $\varphi$ is the homomorphism in (3.16); here $\varphi(\gamma)$ is considered as an element of $K$ using the natural identification of $\widehat{K} \backslash K$ with $K$.

We will show that $\rho_{x, \gamma}^{\prime}$ in (3.18) is independent of the choice of $\gamma$. To prove this, take $\delta:=\gamma \circ b=b \gamma$, where $b \in \pi_{1}(X, x)$. If $T_{\delta}:\left(E_{G}\right)_{x} \longrightarrow$ $\left(E_{G}\right)_{\sigma(x)}$ is the parallel translation, with respect to $\nabla$, along $\delta$, then

$$
T_{\delta}\left(z_{0}\right) \varphi(\delta)=T_{\gamma}\left(z_{0}\right) \varphi\left(b^{-1}\right)(\varphi(b) \varphi(\gamma))=T_{\gamma}\left(z_{0}\right) \varphi(\gamma)
$$

This implies that $\rho_{x, \gamma}^{\prime}=\rho_{x, \delta}^{\prime}$.

Note that $\rho_{x, \gamma}^{\prime}$ takes the natural action of $G$ on $\left(E_{G}\right)_{x}$ to the action of $G$ on $\left(E_{G}\right)_{\sigma(x)}$ obtained by twisting, using $\sigma_{G}$, the natural action of $G$ on $\left(E_{G}\right)_{\sigma(x)}$.

Now take any point $y \in X$. We will construct an isomorphism of $\left(E_{G}\right)_{y}$ with $\left(E_{G}\right)_{\sigma(y)}$.

Take any smooth path $\delta$ from $x$ to $y$. So $\sigma(\delta)$ is a path from $\sigma(x)$ to $\sigma(y)$. Let

$$
T_{\delta}:\left(E_{G}\right)_{x} \longrightarrow\left(E_{G}\right)_{y}
$$

and

$$
T_{\sigma(\delta)}:\left(E_{G}\right)_{\sigma(x)} \longrightarrow\left(E_{G}\right)_{\sigma(y)}
$$

be the parallel translations, with respect to the connection $\nabla$, along $\delta$ and $\sigma(\delta)$ respectively. Let

$$
\rho_{y, \delta}^{\prime}:\left(E_{G}\right)_{y} \longrightarrow\left(E_{G}\right)_{\sigma(y)}
$$

be the map defined by $z \longmapsto T_{\sigma(\delta)} \circ \rho_{x, \gamma}^{\prime} \circ\left(T_{\delta}\right)^{-1}(z)$, where $\rho_{x, \gamma}^{\prime}$ is constructed in (3.18) (we have shown that $\rho_{x, \gamma}^{\prime}$ is independent of $\gamma$ ). 
The above map $\rho_{y, \delta}^{\prime}$ is again independent of the choice of the path $\delta$. To prove this, take $\eta:=\delta \circ b$, where $b \in \pi_{1}(X, x)$. Now

$$
\begin{aligned}
T_{\sigma(\eta)} \circ \rho_{x, \gamma}^{\prime} \circ\left(T_{\eta}\right)^{-1} & =T_{\sigma(\delta)}\left(T_{\sigma(\delta)}\right)^{-1} \circ T_{\sigma(\eta)} \circ T_{\gamma} \varphi(\gamma) \circ \varphi(b)\left(T_{\delta}\right)^{-1} \\
& =T_{\sigma(\delta)} \circ T_{\sigma\left(\delta^{-1} \circ \eta\right) \circ \gamma} \circ \varphi(\gamma b) \circ\left(T_{\delta}\right)^{-1} \\
& =T_{\sigma(\delta)} \circ T_{\sigma(b) \circ \gamma} \circ \varphi(\gamma b) \circ\left(T_{\delta}\right)^{-1} .
\end{aligned}
$$

Therefore, to prove that $\rho_{y, \delta}^{\prime}=\rho_{y, \eta}^{\prime}$, it suffices to show that

$$
T_{\sigma(b) \circ \gamma} \varphi(\gamma b)=\rho_{x, \gamma}^{\prime}
$$

But $\gamma b$ is, by definition, $\sigma(b) \circ \gamma$. Therefore,

$$
T_{\sigma(b) \circ \gamma} \varphi(\gamma b)=\rho_{x, \sigma(b) \circ \gamma}^{\prime},
$$

where $\rho_{x, \sigma(b) \circ \gamma}^{\prime}$ is constructed as in (3.18). But we have seen that $\rho_{x, \gamma}^{\prime}$ is independent of $\gamma$. Therefore, (3.19) holds. Hence $\rho_{y, \delta}^{\prime}$ is independent of the choice of $\delta$.

Using the identification of the total spaces of $E_{G}$ and $\sigma^{*} \bar{E}_{G}$ (see Remark $2.2)$, the above maps $\rho_{y}^{\prime}:=\rho_{y, \delta}^{\prime}, y \in X$, together define a map

$$
\rho: E_{G} \longrightarrow \sigma^{*} \bar{E}_{G}
$$

It can be checked that the pair $\left(E_{G}, \rho\right)$ is a real principal $G$-bundle. In view of Proposition 3.2, the real principal $G$-bundle $\left(E_{G}, \rho\right)$ is polystable because $E_{G}$ is polystable.

It should be clarified that Theorem 3.4 is not true if the assumption that $G$ is semisimple is removed.

\section{Moduli space of principal bundles}

In this section, it is assumed that $G$ is reductive. We also assume that $\operatorname{genus}(X) \geq 3$.

Topological isomorphism classes of principal $G$-bundles over $X$ are parameterized by the fundamental group $\pi_{1}(G)$; any principal $G$-bundle is topologically trivial both on $X \backslash\{x\}$ and on a neighborhood $D$ of $x$, and 
the resulting map $D \backslash\{x\} \longrightarrow G$ produces an element of $\pi_{1}(G)$. Fix a topological isomorphism class

$$
\lambda \in \pi_{1}(G) .
$$

Let $\mathcal{M}_{X}(G)$ be the moduli space of stable principal $G$-bundles on $X$ of the given topological type $\lambda$; see [12] for the construction of $\mathcal{M}_{X}(G)$. This moduli space is a normal quasiprojective complex variety; its dimension is $\operatorname{dim} G(\operatorname{genus}(X)-1)+\operatorname{dim} Z$, where $Z$, as before, is the center of $G$.

Let $E_{G}$ be a holomorphic principal $G$-bundle on $X$, and let $Q$ be a parabolic subgroup of $G$. There is a natural bijective correspondence between the holomorphic reductions of structure group of $E_{G}$ to $Q$ and the holomorphic reductions of structure group of $\sigma^{*} \bar{E}_{G}$ to the parabolic subgroup $\sigma_{G}(Q)$. The identification between the total spaces of $E_{G}$ and $\sigma^{*} \bar{E}_{G}$ (see Remark 2.2) takes the total space of a reduction of structure group of $E_{G}$ to $Q$ to the total space of the corresponding reduction of structure group of $\sigma^{*} \bar{E}_{G}$ to $\sigma_{G}(Q)$. Using this correspondence between reductions of structure group it follows immediately that $E_{G}$ is stable if and only if $\sigma^{*} \bar{E}_{G}$ is stable.

We fix the topological isomorphism class $\lambda$ such that $\sigma^{*} \bar{E}_{G}$ is topologically isomorphic to $E_{G}$ for $E_{G} \in \mathcal{M}_{X}(G)$. Let

$$
\eta: \mathcal{M}_{X}(G) \longrightarrow \mathcal{M}_{X}(G)
$$

be the anti-holomorphic involution defined by $E_{G} \longmapsto \sigma^{*} \bar{E}_{G}$.

A holomorphic principal $G$-bundle $E_{G}$ is said to admit a pseudo-real structure if there is a holomorphic isomorphism of principal $G$-bundles

$$
\rho: E_{G} \longrightarrow \sigma^{*} \bar{E}_{G}
$$

such that the pair $\left(E_{G}, \rho\right)$ is pseudo-real.

Let

$$
\mathcal{M}_{X}^{s}(G) \subset \mathcal{M}_{X}(G)
$$

be the smooth locus of the variety. The involution $\eta$ in (4.1) preserves $\mathcal{M}_{X}^{s}(G)$.

Theorem 4.1. Take any principal $G$-bundle $E_{G} \in \mathcal{M}_{X}^{s}(G)$. Then this $E_{G}$ is fixed by the involution $\eta$ if and only if $E_{G}$ admits a pseudo-real structure.

Proof. Let $\left(E_{G}, \rho\right)$ be a pseudo-real principal $G$-bundle. Then $\rho$ is an isomorphism of $E_{G}$ with $\sigma^{*} \bar{E}_{G}$. Therefore, if $E_{G} \in \mathcal{M}_{X}^{s}(G)$, then $\eta\left(\left\{E_{G}\right\}\right)=\left\{E_{G}\right\}$. We will now prove the converse. 
For a holomorphic principal $G$-bundle $F_{G}$, we have $Z \subset \operatorname{Aut}\left(F_{G}\right)$ (see $(2.5))$. A stable principal $G$-bundle $F_{G}$ on $X$ is called regularly stable if $Z=\operatorname{Aut}\left(F_{G}\right)$. Note that any stable principal $\operatorname{SL}(n, \mathbb{C})$-bundle is regularly stable.

The smooth locus $\mathcal{M}_{X}^{s}(G)$ coincides with the locus in $\mathcal{M}_{X}(G)$ of regularly stable principal $G$-bundles [4, Corollary 3.4]; the assumption that $g \geq 3$ is needed here (note that the moduli space of principal $\mathrm{SL}(2, \mathbb{C})$-bundles on a curve of genus two is smooth as it is isomorphic to $\left.\mathbb{C P}^{3}\right)$.

Let $E_{G} \in \mathcal{M}_{X}(G)$ be a regularly stable principal $G$-bundle such that $\eta\left(\left\{E_{G}\right\}\right)=\left\{E_{G}\right\}$. Fix a holomorphic isomorphism

$$
\theta: E_{G} \longrightarrow \sigma^{*} \bar{E}_{G}
$$

Consider

$$
\sigma^{*} \bar{\theta}: \sigma^{*} \bar{E}_{G} \longrightarrow \sigma^{*} \bar{\sigma}^{*} \bar{E}_{G}=\sigma^{*} \sigma^{*} \overline{\bar{E}}_{G}=E_{G}
$$

as in Definition 2.3. The composition $\left(\sigma^{*} \bar{\theta}\right) \circ \theta$ is a holomorphic automorphism of $E_{G}$. Since $E_{G}$ is regularly stable, there is an element

$$
z_{0} \in Z
$$

such that $\left(\sigma^{*} \bar{\theta}\right) \circ \theta$ coincides with the action of $z_{0}$ on $E_{G}$.

It is straightforward to check that $\theta$ commutes with the composition $\left(\sigma^{*} \bar{\theta}\right) \circ \theta$. Indeed, in terms of the identification of the total space of $E_{G}$ with that of $\sigma^{*} \bar{E}_{G}$ (see Remark 2.2), the map $\sigma^{*} \bar{\theta}$ coincides with $\theta$; this immediately implies that $\left(\sigma^{*} \bar{\theta}\right) \circ \theta$ commutes with $\theta$. Since $\theta$ commutes with $\left(\sigma^{*} \bar{\theta}\right) \circ \theta$, and $\theta$ is an isomorphism between $E_{G}$ and $\sigma^{*} \bar{E}_{G}$, from the construction of $\bar{E}_{G}$ it follows immediately that

$$
\sigma_{G}\left(z_{0}\right)=z_{0}
$$

where $z_{0}$ is the element in (4.2) (recall that $\bar{E}_{G}$ is obtained from $E_{G}$ by twisting the action of $G$ on $E_{G}$ by $\left.\sigma_{G}\right)$. Therefore, $\left(E_{G}, \theta\right)$ is pseudo-real. This completes the proof.

Remark 4.2. The proof of Theorem 4.1 shows that a regularly stable principal $G$-bundle is fixed by the involution $\eta$ if and only if $E_{G}$ admits a pseudoreal structure. Note that this statement is valid even if the genus of $X$ is two. 


\section{Acknowledgments}

We are very grateful to the referee for detailed comments. This work was carried out while both the authors were visiting the Issac Newton Institute. We thank Issac Newton Institute for its hospitality.

\section{References}

[1] B. Anchouche, H. Azad and I. Biswas, Harder-Narasimhan reduction for principal bundles over a compact Kähler manifold, Math. Ann. 323 (2002), 693-712.

[2] B. Anchouche and I. Biswas, Einstein-Hermitian connections on polystable principal bundles over a compact Kähler manifold, Amer. J. Math. 123 (2001), 207-228.

[3] K.A. Behrend, Semi-stability of reductive group schemes over curves, Math. Ann. 301 (1995), 281-305.

[4] I. Biswas and N. Hoffmann, A Torelli theorem for moduli spaces of principal bundles over a curve, Ann. Inst. Fourier 62 (2012), 87-106.

[5] I. Biswas, O. García-Prada and J. Hurtubise, Pseudo-real principal Higgs bundles on compact Kähler manifolds, http://arxiv. org/abs/1209.5814.

[6] I. Biswas, J. Huisman and J. Hurtubise, The moduli space of stable vector bundles over a real algebraic curve, Math. Ann. 347 (2010), 201233.

[7] D. Huybrechts and M. Lehn, The geometry of moduli spaces of sheaves, Aspects Math., E31, Friedr. Vieweg \& Sohn, Braunschweig, 1997.

[8] D. Hyeon and D. Murphy, Note on the stability of principal bundles, Proc. Amer. Math. Soc. 132 (2004), 2205-2213.

[9] S. Kobayashi, Differential Geometry of Complex Vector Bundles, Publications of the Mathematical Society of Japan, 15, Iwanami Shoten Publishers and Princeton University Press, 1987.

[10] S. Ramanan and A. Ramanathan, Some remarks on the instability flag, Tôhoku Math. J. 36 (1984), 269-291.

[11] A. Ramanathan, Stable principal bundles on a compact Riemann surface, Math. Ann. 213 (1975), 129-152. 
[12] A. Ramanathan, Moduli for principal bundles over algebraic curves: II, Proc. Indian Acad. Sci. Math. Sci. 106 (1996), 421-449.

School of Mathematics

Tata Institute of Fundamental Research

HOMI BHABHA ROAD

BOMBAY 400005

INDIA

E-mail address: indranil@math.tifr.res.in

Department of Mathematics

MCGILL UNIVERSITY

Burnside Hall, 805 Sherbrooke St. W., Montreal, Que. H3A 2K6

CANADA

E-mail address: jacques.hurtubise@mcgill.ca

Received August 1, 2011 